\title{
LA HIPNOSIS EN EL TRATAMIENTO DEL DOLOR CRÓNICO
}

\author{
Damaris Muñoz i Cano \\ damarismunoz@ub.edu \\ Guillem Feixas i Viaplana \\ gfeixas@ub.edu \\ Facultad de Psicología. Universidad de Barcelona
}

\begin{abstract}
For a long time, hypnosis has been associated with knowledge areas of little apparent scientific validity. Fortunately, in recent years, hypnosis has proved to be a useful technique for intervention in the health area. The area where more studies have been conducted and with greater success is on the treatment of chronic pain. However, some obstacles must yet be overcome: the limited use of hypnosis in the public health services in Spain, and the resources assigned by universities for research and professional training on these techniques are scarce. This paper aims to briefly review the current state of hypnosis in the treatment of chronic pain, and advocates for its integration within a multidisciplinary treatment of chronic pain.
\end{abstract}

Key words: hypnosis, chronic pain treatment

Tradicionalmente la hipnosis ha sido criticada por parecer poco científica y asociarse a prácticas esotéricas o de espectáculo. En los últimos años se han realizado estudios empíricos que demuestran que la hipnosis tiene una influencia neurofisiológica (De Pascalis, Cacace y Massicolle, 2004; Horton, Crawforf, Harrington y Downs, 2004; Sala 2003) y un efecto positivo en el tratamiento del dolor (Barber, 1996; Barber, 1990; Capafons, 2001; Hilgard y Hilgard, 1975; Montgomery y Schnur, 2005; Patterson y Jensen, 2003; Sala, 2005; Spanos, 1989) y de otras patologías.

La American Psychological Association (APA) en 1993 define la hipnosis como "un conjunto de procedimientos que generan un contexto donde se facilita el cumplimiento de las sugestiones en ciertas personas". Montgomery y Schnur (2005) cuando definen el término matizan que "el paciente puede experimentar cambios en una sensación (por ejemplo, alivio del dolor), en una percepción (por ejemplo, alucinación visual), en una cognición (por ejemplo, expectativas) o en el 
control sobre una conducta motora (por ejemplo, levitación del brazo)". Estos mismos autores, señalan seis mitos en relación a la hipnosis: el primer mito es que el papel del paciente es pasivo. El segundo mito, es que las personas bajo hipnosis pueden perder el control sobre sus acciones. El tercero es que la persona se sentirá diferente. El cuarto es que la persona no podrá salir del trance cuando lo desee. El quinto mito es que los pacientes tendrán amnesia post-hipnótica después de una sesión y finalmente, el sexto es que la hipnosis sólo funciona en pacientes con elevada sugestionabilidad.

Por lo tanto, previamente a iniciar la primera sesión de hipnosis es imprescindible presentar a los pacientes la técnica a emplear. Según Montgomery y Schnur (2005) es necesario educar al paciente en la hipnosis, eliminar los mitos y posibles miedos en relación a la técnica y exponer el grado de eficacia de la misma.

Gracias éstas y a otras muchas aportaciones se ha ido redescubriendo el uso de la hipnosis como una técnica terapéutica amplificadora de la eficacia de otros tratamientos, principalmente farmacológicos (Capafons, 2001; Catel et al., 2007).

El dolor es considerado como el resultado de la interacción de componentes sensoriales, cognitivos y afectivos (Hofstadt y Quiles, 2001). Concretamente, el componente psicológico parece influir en las respuestas fisiológicas y en las estrategias de afrontamiento frente a la situación de dolor (Donker, 1999). El dolor una vez que persiste y se cronifica conlleva un alto grado de malestar en términos de la ansiedad asociada a actividades que pueden aumentar el dolor. El que la vida del paciente gire, en gran medida, en torno al dolor y produzca un grado notable de incapacitación y de disminución de la actividad del paciente es uno de sus efectos más negativos (Soler et al. 2006; Vallejo 2005). Diversos estudios muestran diferencias significativas en las repercusiones sobre el estado de ánimo que presentan personas que padecen dolor crónico respecto a otros con diferente tipo de afecciones (Crombez y cols., 1999; Mccracken y Gross, 1993; Moix, 2004; Turk y Okifuji, 2002). En el ámbito de la psicología de la salud, el tratamiento del dolor crónico es pionero y ha conseguido un impacto positivo sobre la calidad de vida de la persona con dolor crónico.

Los meta-análisis realizados sobre este tema demuestran que la adición de la hipnosis a los tratamientos habituales mejora considerablemente su eficacia (Kirsch, Montgomery y Sapirstein, 1995; Montgomery, Duhamel y Redd, 2000). En un trabajo realizado por Montgomery et al. (2000) que un $75 \%$ de los pacientes se beneficiaron respecto al grupo control en el uso de la hipnosis como tratamiento del dolor.

En el campo donde quizás más se ha comprobado la eficacia de la hipnosis es en el tratamiento del dolor, conociéndola como hipnoanalgesia. En un estudio de Castel et. al, (2007) con 45 pacientes con diagnóstico de fibromialgia se compararon tres condiciones experimentales. La primera, hipnosis con sugestiones de relajación. La segunda, hipnosis con sugestión analgésica y finalmente, una tercera con 
relajación. Así pues, se presentaron las dos primeras condiciones experimentales como hipnosis. Los resultados indican que la hipnosis con sugestiones analgésicas tiene más efecto en la intensidad del dolor y en la dimensión sensorial del mismo que la hipnosis con sugestión de relajación. Y que el efecto de la hipnosis con sugestiones de relajación no es mayor que el de la relajación.

El hecho de que las sugestiones de analgesia presentaran mayores cambios en la intensidad del dolor y en los componentes sensoriales del dolor manifiesta que el contenido de la sugestión es importante e indican que algunas sugestiones son más efectivas que otras en el control del dolor.

Como afirman los autores, definir las diferencias entre hipnosis y otros métodos para inducir relajación es un trabajo complicado porque todos contienen componentes de relajación y de focalización de la atención y además, parece que no se han comprobado sus diferencias empíricas (Syrjala y Abrams, 1996).

En el estudio de Castel et al, (2007) la condición experimental de hipnosis con sugestiones de relajación difiere de la condición experimental de relajación en que a los participantes de hipnosis se les pidió que visualizaran una imagen y a los de la condición de relajación que se centraran en las sensaciones corporales de relajación y respiración. Castel et al. (2007) consideran que la ausencia de diferencias en los resultados de ambas condiciones se deben a que ambas utilizan sugestiones exclusivamente de bienestar y confort, es decir, un componente afectivo del dolor.

Por otro lado, se ha comprobado que existen correlatos fisiológicos de la hipnosis clínica sobre el paciente a través de técnicas de neuroimagen (SPECT, PET) y de electrofisiología (EMG, EEG, PEC, etc) (Sala, 2005).

Según Moix(2002) el uso de la hipnosis como técnica para reducir el dolor crónico (cefaleas, fibromialgia, dolor lumbar,...) es empleada habitualmente dentro del tratamiento cognitivo - conductual con el objetivo de reducir el dolor, la medicación, las respuestas emocionales de ansiedad y depresión, y finalmente, aumentar la actividad física. Es decir, que los pacientes aprendan a responder a las sugestiones y posteriormente puedan aplicar esa sensación de analgesia no estando bajo hipnosis. No obstante, no está claro cuál es el mecanismo de la hipnosis que influye en disminuir el dolor.

En un trabajo sobre la hipnosis en el tratamiento del dolor Moix (2002) expone tres hipótesis que justifican la evidencia de disminución del dolor crónico mediante la hipnosis, pero que ninguna de ellas por sí sola resulta una explicación satisfactoria. La primera hipótesis sostiene que la hipnosis disminuye el dolor mediante la reducción de la ansiedad. No obstante, esta hipótesis no parece sustentarse principalmente en procesos muy dolorosos como puede ser una intervención quirúrgica $\sin$ anestesia. La segunda, las creencias, parece ser que la palabra hipnosis aumenta la expectativa respecto a la eficacia del resultado del tratamiento. Y finalmente, la redirección de la atención. Existen hipótesis que sustentan que mediante la hipnosis 
los estímulos dolorosos se procesan a nivel inconsciente y que las sugestiones se procesan a nivel consciente.

La prohibición de la hipnosis en la sanidad pública (BOE 10/2/94) y los pocos recursos en el contexto universitario en la formación de la hipnosis conlleva que en España existan pocas investigaciones sobre la hipnosis (Capafons, 2002b; Vallejo y Capafons, 2005). Con respecto a lo primero, en la Universitat de Barcelona existe desde el 2004 un posgrado en Hipnosis Clínica. Ello constituye un buen paso en la dirección de mejorar la formación en hipnosis de los profesionales. Hay que tener en cuenta que la falta de formación y el mal uso de la hipnosis puede conducir a una cierta iatrogenia (Capafons, 2005). En relación a la investigación, parece que empieza a desarrollarse estudios interesantes y competitivos (p. ej., Castel et al. 2007).

Según Sala (2003, Abril) todavía quedan dos frentes abiertos. Por un lado, la Administración, ya que el tiempo que se emplea en una sesión de hipnosis es considerablemente superior al que la Administración estipula por paciente. Y el otro frente abierto es el de la industria farmacéutica, ya que la efectividad de la hipnosis podría influir en una reducción del uso de fármacos en el tratamiento del dolor crónico.

Montgomery y Schnur (2005) consideran 3 áreas de investigación importantes a desarrollar sobre el uso de la hipnosis: estudios de los cambios cerebrales asociados a la hipnosis, estudios de los mecanismos psicológicos de los efectos hipnóticos y finalmente, estudios de costo - beneficio.

Asimismo, es necesario avanzar en definir el tratamiento más idóneo para el perfil y necesidades específicas de cada paciente. Esto resulta de mayor importancia en el dolor crónico, ya que como anteriormente hemos citado, los pacientes padecen alteraciones en el estado emocional y una elevada comorbilidad con trastornos de ansiedad, problemas de pareja, disfunciones sexuales, etc. (Vallejo, 2005).

Pero a la vista de los resultados expuestos y de otras investigaciones en este campo estamos de acuerdo con Barber (2000) en diferenciar los beneficios que la hipnosis por sí sola puede proporcionar al paciente y el uso de la hipnosis integrada en el tratamiento psicológico multi-componente del dolor crónico. Que se obtengan beneficios que perduren en el tiempo es más probable a través de una intervención interdisciplinar en el que se incluyan talleres psicoeducativos sobre la patología para pacientes y familiares, intervención psicológica familiar e individual con el uso de técnicas terapéuticas que se ajusten al paciente y finalmente, un tratamiento médico indicado(Capafons, 2001; Hofstadt y Quilez, 2001; Penzo, 1989; RodríguezMarín y García, 1988). 
Durante mucho tiempo la hipnosis ha sido asociada con áreas de conocimiento que parecen considerarse de poca validez cientifica. Afortunadamente, en los últimos años, el uso de la hipnosis como técnica de intervención en el área de la salud ha demostrado su eficacia. En el campo donde se han realizado mayor numero de estudios y donde se ha obtenido mayor éxito ha sido en el tratamiento del dolor crónico. Aun así, deben superarse algunos obstáculos como su uso muy limitado en la sanidad pública y su escasa presencia en la investigación y la formación universitaria. El presente trabajo pretende realizar una breve revisión teórica sobre la situación actual de la hipnosis en el tratamiento del dolor crónico y apostar por su integración dentro de un tratamiento multidisciplinar.

Palabras clave: hipnosis, tratamiento del dolor crónico

\section{Referencias bibliográficas}

BARBER, J. (2000). Tratamiento del dolor mediante hipnosis y sugestión. Bilbao: Desclée de Brouwer.

BARBER, J. (1990). Hypnosis. En J. Bonica. (Ed.). The management of pain (pp. 1733-1741). PhiladelphiaLondon: Lea/Febiger.

BARBER J. (1996). Hypnosis and suggestion in the treatment of pain. A clinical guide. New York: Norton.

CAPAFONS, A. (2001) Hipnosis. Madrid: Sintesis.

CAPAFONS, A. (2002, Agosto). Hypnopsis in Spain: The long Way to Run. Comunicación presentada en la 110 Annual Convention of the American Psychological Association, Chicago, EEUU.

CAPAFONS, A. (2005). Hipnosis. Papeles del Psicólogo, 89, 1-2.

CASTEL, A., PÉREZ, M., SALA, J., PADROL, A. y RULL M. (2007). Effect of hypnotic suggestion on fibromyalgic pain: Comparison between hypnosis and relaxation. European Journal of Pain, 11, 463-468.

CHAVES, J.F. (1994). Recent advances in the application of hypnosis to pain management. American Journal of Clinical Hypnosis, 43, 17-40.

CROMBEZ, G., VLAEYEN, J.W. y HEUTS, P.H.(1999). Pain-related fear is more disabling than pain itself: evidence on the role of pain-related fear in chronic back pain disability. Pain, 80, 329-339.

DE PASCALIS, V., CACACE, I. y MASSICOLLE, F. (2004, Noviembre). Perception and modulation of pain in waking and hypnosis: functional significance of phase ordered gamma oscillations. Pain, 12, 27-36.

DONKER, F.J.S. (1999). Tratamiento psicológico del dolor crónico. En Simón MA. Manual de psicología de la Salud. Madrid: Biblioteca Nueva, 537-556.

EIMER, B.N. (2000). Clinical applications of hypnosis for brief and efficient pain management psychotherapy. American Journal of Clinical Hypnosis, 43, 17-40.

HILGARD, E.R. y HILGARD, J.R. (1975). Hypnosis in the relief of pain. Los Altos, CA: William Kaufmann.

VAN-DER HOFSTADT, C.J. y QUILES, M.J. (2001). Dolor crónico: intervención terapéutica desde la psicología. Rev. Soc. Esp. Dolor, 8, 503-510.

HORTON, J.E., CRAWFORD, H.J., HARRINGTON, G. y DOWNS, J.H. (2004). Increased anterior corpus callosum size associated positively with hypnotizability and the ability to control pain. Brain, 127, 1741-7.

KIRSCH, I., MONTGOMERY, G. y SAPIRSTEN, G. (1995). Hypnosis as an adjunct to cognitive-behavioral psychotherapy: a meta-analysis. J Consult Clin Psychol, 63, 214-20.

LYNN, S.J. y KIRSCH, I. (2005). Teorías de hipnosis. Papeles del psicólogo, 89, 9- 15.

MCCRACKEN,L.M. y GROSS,R.T.(1993). Does anxiety affect coping with chronic pain?

Clinical Journal of Pain, 9, 253-259.

MOIX, J. (2002). La hipnosis en el tratamiento del dolor. Revista Sociedad Española del Dolor, 9, 525-532.

MONTGOMERY, G.H., DUHAMEL, K.N. y REDD, W.H. (2000). A meta-analysis of hypnotically induced analgesia: how effective is hypnosis? International Journal of Clincical and Experimental Hypnosis, 48, $138-153$.

MONTGOMERY, G.H.y SCHNUR, J.B. (2005). Eficacia y aplicación de la hipnosis clínica. Papeles del Psicólogo, 89, 3-8. 
PATTERSON, D.R. y JENSEN, M.P.(2003). Hypnosis and clinical pain. Psychological Bulletin, 129, 495-521. PENZO, W. (1989). El dolor crónico. Aspectos psicológicos. Barcelona: Martínez Roca.

RODRÍGUEZ-MARÍN, J. y GARCÍA, J.A. (1988). Aplicación de técnicas cognitivo-comportamentales en un caso de dolor crónico. En: Macià D. y Méndez F.X., (eds.), Aplicaciones clínicas de la evaluación y modificación de conducta. Estudio de casos (pp. 309-338). Madrid: Pirámide.

SALA, J. (2003). Hipnosis en el tratamiento del dolor. Medicina Clínica, 121, 219-220.

SALA, J. (2003, Abril). Investigación Cientifica de la hipnosis. Conferencia. Asociación Española de Hipnosis Ericksoniana. Artículo obtenido el 2 de Diciembre de 2007 en http://www.aehe.com/ultima_hora2.htm

SALA, J. (2005, Octubre). Tractament del dolor fibromiàlgic amb hipnosis i fàrmacs vs. sols fàrmacs. Sessió Clínica de la Societat Catalana d'Anastesiologia, Reanimació i Terapèutica del Dolor, Acadèmia de Cièncias Mèdiques de Catalunya i Balears.

SYRJALA, K.L. y ABRAMS, J.R. (1996). Hypnosis and imagery in the treatment of pain. In: Gatchel, RJ, Turk, D.C. (Eds.). Psychological approaches to pain management: a practitioner's handbook (pp. 231-258). New York: Guilford

SOLER, MD., SAURÍ, J., ROMERO, A., OCHOA, M., VIDAL, J., CURCOLL, LL y MOIXJ. (2006). Tratamiento interdisciplinar del dolor crónico en pacientes lesionados medulares. Resultados preliminares a propósito de tres casos. Dolor, 21, 219-230.

SPANOS N.P., PERLINI A.H. y ROBERTSON L.A. (1989). Hypnosis, suggestion and placebo in the reduction of experimental pain. Journal of Abnormal Psychology, 205, 285-293.

TUNER, J.A. y CHAPMAN,C.R.(1982). Psychological interventions for chronic pain: a critical review. II. Operant conditioning, hypnosis, and cognitive-behavioral therapy. Pain, 12, 23-46.

TURK,D.C. y OKIFUJI,A.(2002). Psychological factors in chronic pain: evolution and

revolution. Pain, 70, 678-690.

VALLEJO, M.A. (2005) Tratamiento psicológico del dolor crónico. Boletín de Psicología, 84, 41-58.

VALLEJO, M.A. y CAPAFONS, A. (2005). La hipnosis psicológica en España. Papeles del Psicólogo, 89, 39-43. 